\title{
Propagación in situ de ratania - Krameria lappacea (Krameriaceae): factores limitantes de la propagación natural y efectos de resiembra
}

\section{In situ propagation of rhatany - Krameria lappacea (Krameriaceae): factors limiting natural re- generation and effects of reseeding measures}

\author{
Nicolas Dostert ${ }^{1}$, Fatima Caceres* ${ }^{2}$, Grischa Brokamp ${ }^{3}$ y Maximilian Weigend ${ }^{4}$
}

1 botconsult $\mathrm{GmbH}$, Muehlenweg 3, 88633 Heiligenberg, Alemania.

2 Herbarium areqvipense HUSA. Departamento de Biología. Universidad Nacional de San Agustín. Av. Daniel Alcides Carrión s/n. La Pampilla. Arequipa.

3 Botanischer Garten Botanisches Museum Berlin, Freie Universität Berlin. Königin-Luise-Straße 6-8, D-14195 Berlin, Alemania.

4 Nees-Institut für Biodiversität der Pflanzen, Rheinische Friedrich-Wilhelms-Universität, Meckenheimer Allee 170, D-53115 Bonn, Alemania.

*Autor de correspondencia

Email Nicolas Dostert: dostert@botconsult.de

Email Fatima Caceres: dcaceresh@unsa.edu.pe

Email Grischa Brokamp: g.brokamp@bgbm.org

Email Maximilian Weigend: mweigend@uni-bonn.de

\begin{abstract}
Resumen
Krameria lappacea (ratania) ha sido utilizada y reconocida como planta medicinal y tintura vegetal desde épocas precolombinas. En los últimos 15 años se han estudiado la biología, propagación y abundancia de ratania en Perú y desarrollado métodos que garanticen una recolección silvestre sostenible. El objetivo de este trabajo es investigar qué factores limitan la propagación natural y evaluar si una resiembra de semillas durante la recolección puede facilitar el establecimiento de plantas nuevas in situ. Al investigarse la germinación de las semillas y la propagación in situ pudo comprobarse mediante experimentos de invernadero que el entierro de las semillas es un factor limitante para la germinación de $K$. lappacea y que la tasa de germinación puede incrementarse significativamente $(3-4 \mathrm{x})$ al enterrar las semillas. Tanto la resiembra de las semillas en los hoyos realizados tras la cosecha, así como la resiembra más esparcida en parcelas seleccionadas, puede contribuir al reforzamiento de la regeneración natural. Las raíces secundarias que quedan en el suelo después de la cosecha forman un nuevo meristemo y dan lugar al desarrollo de nuevas plantas. El pre-tratamiento de las semillas (escarificación) no produce ningún efecto claro en el éxito de la germinación, sin embargo, esta práctica facilita la manipulación durante la resiembra, asegurando que únicamente semillas viables y completamente desarrolladas sean utilizadas posteriormente.
\end{abstract}

Palabras clave: Krameria lappacea; recolección silvestre; germinación; tratamiento de semillas; manejo sostenible.

\section{Citación:}

Dostert N., F. Caceres, G. Brokamp y M. Weigend. 2018. Propagación in situ de ratania - Krameria lappacea (Krameriaceae): factores limitantes de la propagación natural y efectos de resiembra. Revista peruana de biología 25(1): 029 - 034 (Febrero 2018). doi: http://dx.doi.org/10.15381/ rpb.v25i1.14345

Fuentes de financiamiento: El presente trabajo se realizó gracias al apoyo de la empresa WELEDAAG Arlesheim, Suiza, y la Sociedad Alemana de Cooperación Internacional (GIZ) GmbH, Eschborn, Alemania, en el marco de cooperación interinstitucional público-privada 2002-2007 (recolección silvestre sostenible, conservación del hábitat y cultivo de la planta andina amenazada ratania: VN 81060590)

\section{Información sobre los autores:}

MW, ND, diseño de la presente investigación. FC, resiembra y evaluación de plantas en el campo. MW y GB germinación de semillas y "entierro de semillas" (seed burial). FC, MW, ND colecta e identificación de las muestras botánicas. ND, FC realizó observaciones de raíces secundarias. ND la redacción de gran parte del presente manuscrito. Todos los autores revisaron y aprobaron el manuscrito.

Presentado: $\quad 13 / 07 / 2017$

Aceptado: $\quad 20 / 01 / 2018$

Publicado online: $27 / 02 / 2018$ 


\begin{abstract}
Krameria lappacea (rhatany) is a well established medicinal and dye plant that is used since pre-Colombian times. In the last 15 years the biology, distribution and abundance of rhatany in Peru was investigated and methods for a sustainable wildcrafting were developed. The objective of this work was to investigate which factors limit the natural propagation and to explore whether the reseeding measures can contribute to the establishment of new plants in situ. Investigating the germination and in situ propagation, greenhouse trials showed that the seed burial is an important, limiting factor for the germination of $K$. lappacea und the germination rate can be increased $(3-4 x)$ by burying the seeds. Both the burial of the seeds during collection and re-sowing measures on selected lots can contribute to the rejuvenation of the population. Secondary roots, which are left in the ground during harvest, are showed to form new shoot meristems and develop into new plants. Pre-treatment (scarification) of the fruits does not clearly influence the germination success, but it facilitates the handling of the seeds during re-sowing and ensures the use of only fully developed, viable seeds.
\end{abstract}

Keywords: Krameria lappacea; wildcrafting; germination; seed treatment; sustainable use.

\section{Introducción}

Ratania [Krameria lappacea (Dombey) Burdet \& Simpson, Krameriaceae, sin. Krameria triandra Ruíz \& Pavon] es un arbusto que se distribuye en los semidesiertos de los Andes peruanos, así como en el sur de Ecuador, el norte de Argentina, Chile y Bolivia (Simpson 2004, 2007). El género Krameria se encuentra principalmente en zonas de vegetación abierta y estacional-seca, algunas especies se distribuyen también en hábitats templados en Norte y Sudamérica.

La raíz de ratania es utilizada en la medicina popular peruana tanto como planta medicinal como tintura vegetal, debido a su contenido de taninos (Simpson 1989, 1991). Actualmente la raíz de ratania es utilizada en preparaciones farmacéuticas y cosméticas, especialmente en productos para el cuidado dental (Adwan et al. 2012). Las raíces contienen $8-18 \%$ de taninos de ratania y la corteza de la raíz hasta un $40 \%$. Adicionalmente, las raíces también contienen neolignanos (Scholz \& Rimpler 1989, Carini et al. 2002).

El hábito típico de $K$. lappacea es un arbusto decumbente a erecto con una altura de $30-80(-100) \mathrm{cm}$ y un diámetro de hasta $1.5(-3) \mathrm{m}$. La morfología de la flor es una de las características más llamativas de las Krameriaceae (Fig. 2A). Aunque las flores oleíferas y zigomórficas de Krameria son visitadas por las abejas hembras del genero Centris, especializadas en colectar el aceite de las flores que parecen ser fácilmente fertilizadas en la naturaleza (Kuijt 1969, Simpson 1982). El fruto de Krameria es una nuez con una sola semilla. La semilla permanece encerrada dentro de la cubierta dura con pelos espinosos rojizos (Fig. 2B). Por lo que en el texto nos referiremos al fruto con la semilla como "semilla" para evitar la confusión.

Entre los años 2002 - 2007, dentro del marco de la cooperación interinstitucional público-privada entre WELEDA AG y la Asociación GTZ (actualmente GIZ o Sociedad Alemana de Cooperación Internacional) se inició un complejo proyecto para investigar la biología, propagación y abundancia de ratania en Perú, y para desarrollar métodos que aseguren la recolección silvestre sostenible (Weigend \& Dostert 2005, 2008). El objetivo era sustituir la recolección silvestre depredadora existente por una recolección sostenible, basada en un plan de acción apoyado en bases científicas y con una cadena de valor transparente. Estos estudios se continúan hasta el día de hoy, para guiar la recolección silvestre comercial.
Como parte de este proyecto se pudo demostrar que $K$. lappacea es, al igual que las demás especies del género Krameria, una planta hemiparásita que incluso fotosintetiza por sí misma, pero que obtiene nutrientes minerales de las raíces de las plantas hospederas a través de haustorios, como una parásita del xilema (Cannon 1910, Musselman 1975, Brokamp et al. 2012). A pesar del número relativamente limitado de especies perennes en el hábitat estacionalmente seco de ratania, se pudo demostrar que $K$. lappacea establece conexiones con las raíces de 18 especies diferentes, pertenecientes a 17 géneros y 12 familias de plantas. Krameria lappacea es por lo tanto una hemiparásita obligada generalista.

En varias especies de plantas, particularmente en hábitats áridos, se ha demostrado que el llamado entierro de semillas" (seed burial) incrementa significativamente la tasa de germinación de las semillas (Beck 2010, Ren 2002). El seed burial se refiere a la siembra natural de las semillas bajo tierra, ya sea por medio de movimientos del suelo o por animales (roedores, scatter hoarding). También para $K$. lappacea se ha demostrado que al enterrar las semillas se aumenta significativamente la tasa de germinación (Brokamp 2015).

Con la finalidad de investigar la frecuencia y propagación se realizaron inventarios en 238 áreas pertenecientes a 19 localidades en seis departamentos del Perú (Brokamp 2015). La abundancia promedio de todos los 19 sitios fue de aproximadamente 14 individuos $/ 100 \mathrm{~m}^{2}$, aunque se encontraron grandes diferencias entre sitios. Se pudo demostrar que la presión de la recolección así como los factores bióticos y abióticos influyen en el desarrollo de las poblaciones. Los métodos de recolección no sostenibles al igual que la escasez en las precipitaciones, es decir, períodos lluviosos cortos que no son suficientes para el establecimiento exitoso de las plántulas, afectan el tamaño de las poblaciones de forma negativa.

Sobre la base de los resultados del proyecto se desarrolló una estrategia para la recolección silvestre sostenible fundamentada en dos principios básicos, los cuales minimizan el impacto inmediato de la cosecha, reemplazando todas las plantas cosechadas a mediano plazo y conllevando incluso a un incremento en el número de plantas (Weigend \& Dostert 2008). En primer lugar, solamente un pequeño porcentaje bien definido de las plantas aptas para cosechar serán recolectadas cada año (una de cinco) y los terrenos serán usados para la recolección silvestre únicamente en 
intervalos de 10 años. En segundo lugar, después de la cosecha, todas las semillas que quedan en el suelo serán sembradas en los hoyos, de tal forma que allí puedan desarrollarse nuevas plantas.

La mayoría de las raíces de ratania comercializadas en el mercado mundial provienen de recolectas silvestres realizadas en Perú (Weigend \& Dostert 2005). La recolección silvestre con fines comerciales en otros países del área de distribución es probablemente imposible a causa de la baja abundancia. El cultivo de ratania es hasta ahora desconocido debido a las dificultades asociadas al hemiparasitismo y al largo período de crecimiento.

El objetivo principal de este trabajo es investigar qué factores limitan la regeneración natural. Específicamente la investigación sobre la viabilidad de las semillas y experimentos del entierro de semillas (seed burial) permiten la determinación de estos factores. El segundo objetivo es evaluar si una resiembra de semillas durante la recolección puede facilitar el establecimiento de plantas nuevas in situ y dar lugar a la regeneración natural. La resiembra en este contexto describe el proceso de enterrar semillas en el hábitat natural de la ratania. Específicamente la evaluación de la emergencia de las semillas y el crecimiento de las plántulas in situ puede mostrar el logro de estas medidas.

\section{Material y métodos}

Evaluación de la viabilidad y germinación de semillas.- Las semillas de $K$. lappacea usadas para la evaluación de la viabilidad, imbibición y germinación fueron colectadas el 13 de agosto de 2003 en San Antonio, Yarabamba, Dpto. Arequipa (GPS $16^{\circ} 34.411^{\prime} \mathrm{S}, 071^{\circ} 28.456^{\prime} \mathrm{W}$, espécimen referencia: N. Dostert 1013). Para la determinación de la viabilidad se utilizaron 40 semillas a las cuales se les retiró la cubierta exponiendo de esta forma el embrión. Este fue cortado por la mitad y fue sumergido en una solución de trifeniltetrazol $0.1 \%$ e incubado durante 24 horas en la oscuridad. Después de esto se determinó la viabilidad de los embriones. Para la determinación de la imbibición y la tasa de germinación se utilizaron 40 semillas cuya cubierta fue quebrada con un martillo. Después de esto se colocaron 40 semillas sin tratamiento y con la cubierta quebrada, respectivamente, en un plato Petri con agua y se dejaron en remojo durante 24 horas. Posteriormente se determinó la tasa de imbibición y se sembraron las semillas germinadas libres de la cubierta dura del pericarpo, en macetas de plástico. Después de 21 días se determinó la tasa de germinación de las semillas.

Germinación de semillas y "entierro de semillas" (seed burial).- Para investigar el seed burial se colectó una gran cantidad de semillas maduras de $K$. lappacea completamente desarrolladas en abril del 2009 en Omate, Dept. Moquegua (16³9.445'S, $071^{\circ} 00.046^{\prime} \mathrm{W} 2500 \mathrm{~m}$ de altitud, espécimen referencia: M. Weigend \& Ch. Schwarzer 7873). En los experimentos fueron utilizadas 600 semillas maduras no tratadas y se establecieron seis lotes de prueba, cada uno con 100 semillas. Se investigaron tres diferentes profundidades de siembra: Sobre la superficie del substrato, a $5 \mathrm{~cm}$ de profundidad y a $10 \mathrm{~cm}$ de profundidad. Se utilizaron para cada tratamiento respectivamente 10 macetas, cada una con 10 semillas. Esta aproximación de $3 \times 10$ macetas $(3 \times 10 \times 10=300$ semillas $)$ se llevó a cabo por duplicado, teniendo un conjunto completo de macetas con semillas no tratadas (tal cual como fueron colectados en la naturaleza) y un segundo conjunto de macetas con semillas que previamente fueron frotados con arena fina, con el fin de debilitar el pericarpio y retirar los pelos gloquidiados. Como substrato se utilizó una mezcla de $50 \%$ compost de tierra tratada al vapor y $50 \%$ arena de río fina, lo cual se asemeja estructuralmente a la composición del suelo en el sitio de colecta silvestre. El riego se realizó únicamente una vez por semana con el fin de evitar el encharcamiento y la alta humedad no fisiológica del suelo. La siembra se llevó a cabo el 8 de octubre de 2009. La temperatura se mantuvo entre $20-27^{\circ} \mathrm{C}$ y la iluminación 12 horas/día. La germinación de las plantas se documentó primero a través del conteo de las plántulas resultantes, y al final del experimento (15 de diciembre de 2009) se analizó el substrato para contar y separar las semillas no germinadas de las germinadas (no visibles sobre la superficie). La cantidad de semillas germinadas en los diferentes tratamientos fue comparada y la diferencia estadísticamente significativa fue calculada mediante las pruebas U de MannWhitney y la $\mathrm{T}$ de significancia.

Evaluación de emergencias de semillas y crecimiento de plántulas in situ.- Las semillas utilizadas en los experimentos de resiembra en las localidades silvestres fueron colectadas en noviembre de 2011 en las laderas de Arequipilla, Chuquibamba, Dpto. Arequipa. La resiembra se llevó a cabo en un área de 200 $\mathrm{m}^{2}$ en las laderas de Huaccllay/Arequipilla (GPS: $15^{\circ} 52.590^{\prime} \mathrm{S}$, $072^{\circ} 38.382^{\prime} \mathrm{W}, 2656$ - $2652 \mathrm{~m}$ de altitud). Se resembraron respectivamente 10 semillas no tratadas en 10 hoyos.

\section{Resultados}

Evaluación de la viabilidad y germinación de semillas.Los experimentos preliminares de la viabilidad de las semillas de $K$. lappacea mostraron que el $75 \%$ de las semillas tratadas con la solución de trifeniltetrazol (0.1\%) fueron viables. El 25\% resultaron ser inviables. La tasa de imbibición y germinación de las semillas después de 21 días pudo ser aumentado a través de la escarificación de la cubierta de las semillas, mejorando consiguientemente la absorción del agua (Fig. 1). Tras el daño mecánico se observó imbibición en el 95\% de las semillas y germinación en el $62 \%$, en las semillas sin daño a la cubierta el porcentaje fue solo del $35 \%$ en ambos casos.

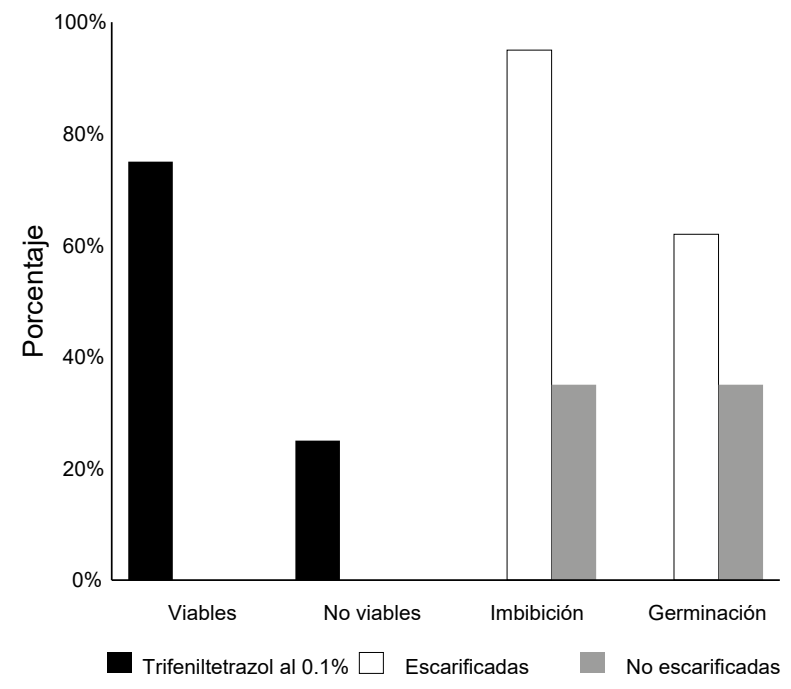

Figura 1. Tasa de viabilidad $(n=40)$, imbibición $(n=40)$ y germinación ( $n=40)$ de las semillas de $K$. Lappacea. 
Germinación de semillas y "entierro de semillas" (seed burial).- Los estudios de campo han demostrado que existe una cantidad excepcional de semillas en las poblaciones naturales de ratania. Las semillas, las cuales son parcialmente estropeadas por la ingestión de los animales, a menudo se encuentran formando grandes grumos, con los pelos gloquidiados enredados entre sí bajo las ramas de los arbustos de las ratanias, siendo exitosamente dispersados de esta forma. De manera equivalente, la gran cantidad de semillas viables presentes en los hábitats naturales contrasta fuertemente con la débil regeneración natural de $K$. lappacea.

El éxito de germinación se encuentra resumido en la Tabla 1. Se obtuvo una tasa de germinación de 1 a 10 semillas por maceta, siendo la tasa de germinación de las semillas no enterradas $(16 \%$ tratadas y $28 \%$ no tratadas) mucho menor que la de las semillas enterradas $(82-88 \%)$. Sin embargo no se encontraron diferencias significativas entre las semillas tratadas y las no tratadas que fueron enterradas a la misma profundidad, al igual que no hubo diferencias significativas entre las semillas sembradas a 5 y a $10 \mathrm{~cm}$ de profundidad. Las semillas no tratadas germinaron ligeramente mejor sobre la superficie del suelo que las tratadas. La Tabla 2 muestra la significancia estadística de las diferentes germinaciones por tratamiento y condiciones de germinación. De ello se deduce que las diferencias estadísticamente significativas se presentaron entre las semillas esparcidas sobre la superficie y las semillas enterradas, pero no entre las profundidades de siembra $(5$ o $10 \mathrm{~cm}$ ) o los pre-tratamientos (semillas no tratadas versus semillas tratadas).
Evaluación de la emergencia de las semillas y del crecimiento de las plántulas in situ.- Las pruebas de siembra en las localidades silvestres mostraron que 66 de las 100 semillas aportadas germinaron después de la primera época de lluvia (Tabla 3). De estas plántulas 30 sobrevivieron al siguiente periodo seco, alcanzaron un tamaño de $3-5 \mathrm{~cm}$ y por consiguiente pudieron establecerse en la localidad silvestre (Fig. 2E). Durante el segundo año las plantas alcanzaron una altura de $6-10 \mathrm{~cm}$ y en el subsecuente, de $17-23$ o $32-37 \mathrm{~cm}$ (Fig. 2F). En el tercer año después de la siembra todas las plantas florecieron por primera vez.

Brote de raíces secundarias.- Un aspecto adicional del manejo sostenible y de la propagación in situ es el rebrote de las raíces secundarias. Los colectores locales han observado desde hace tiempo que las raíces secundarias que permanecen en el suelo después de la cosecha vuelven a brotar. Esta observación pudo ser confirmada en el campo. A partir del cambium de las raíces secundarias remanentes se vuelve a producir un meristemo a partir del cual brotan y crecen nuevas plantas (Fig. 2C, D). Hasta el momento no hay datos sobre la frecuencia de la producción de brotes a partir de la raíz, sin embargo las observaciones en el campo demuestran que este no es un fenómeno aislado.

\section{Discusión}

A diferencia de los estudios de la evaluación de la viabilidad y germinación de semillasno se pudo demostrar en los experimentos de "entierro de semillas" (seed burial) un efecto positivo

Tabla 1. Promedio de germinacion de Krameria lappacea $(\mathrm{U}=$ semilla no tratada, $\mathrm{B}=$ semilla tratada, $\mathrm{cm}$ : profundidad de siembra)

\begin{tabular}{lcccccc}
\hline Nro. & $\mathbf{0 ~} \mathbf{~ m ~} \mathbf{~}$ & $\mathbf{5 ~} \mathbf{~ m ~ \mathbf { ~ }}$ & $\mathbf{1 0} \mathbf{~ c m ~} \mathbf{~}$ & $\mathbf{0 ~} \mathbf{~ m ~ B ~}$ & $\mathbf{5 ~} \mathbf{~ m ~ B ~}$ & $\mathbf{1 0} \mathbf{~ c m ~ B ~}$ \\
\hline $\begin{array}{l}\text { Promedio } \\
\text { \% Germinación (y desviación } \\
\text { estándar) }\end{array}$ & 2.8 & 8.2 & 8.8 & 1.6 & 8.5 & 8.6 \\
\hline
\end{tabular}

Tabla 2. Comparación estadística entre los distintos tratamientos y profundidades de siembra de Krameria lappacea.

\begin{tabular}{|c|c|c|c|c|c|c|}
\hline$n=10$ & $0 \mathrm{~cm} \mathrm{U}$ & $5 \mathrm{~cm} \mathrm{U}$ & $10 \mathrm{~cm} \mathrm{U}$ & $0 \mathrm{~cm} \mathrm{~B}$ & $5 \mathrm{~cm} \mathrm{~B}$ & $10 \mathrm{~cm} \mathrm{~B}$ \\
\hline $0 \mathrm{~cm} \mathrm{U}$ & - & $\begin{array}{l}\mathrm{p}<0.001 \\
\mathrm{t}=-9.823 \\
\mathrm{df}=15.067\end{array}$ & $\begin{array}{l}p<0.001 \\
t=-11.339 \\
d f=13.755\end{array}$ & $\begin{array}{l}p=0.067 \\
t=1.952 \\
d f=17.589\end{array}$ & $\begin{array}{l}\mathrm{p}<0.001 \\
\mathrm{t}=-11.015 \\
\mathrm{df}=12.926\end{array}$ & $\begin{array}{l}\mathrm{p}<0.001 \\
\mathrm{t}=-10.791 \\
\mathrm{df}=14.311\end{array}$ \\
\hline $5 \mathrm{~cm} \mathrm{U}$ & - & - & $\begin{array}{l}p=0.135 \\
t=-1.567 \\
d f=17.596\end{array}$ & $\begin{array}{l}\mathrm{p}<0.001 \\
\mathrm{t}=13.349 \\
\mathrm{df}=16.430\end{array}$ & $\begin{array}{l}\mathrm{p}=0.425 \\
\mathrm{t}=-0.818 \\
\mathrm{df}=16.891\end{array}$ & $\begin{array}{l}\mathrm{p}=0.324 \\
\mathrm{t}=-1.014 \\
\mathrm{df}=17.869\end{array}$ \\
\hline $10 \mathrm{~cm} \mathrm{U}$ & - & - & - & $\begin{array}{l}\mathrm{p}<0.001 \\
\mathrm{t}=15.274 \\
\mathrm{df}=15.080\end{array}$ & $\begin{aligned} \mathrm{p} & =0.382 \\
\mathrm{t} & =0.896 \\
\mathrm{df} & =17.789\end{aligned}$ & $\begin{array}{l}\mathrm{p}=0.591 \\
\mathrm{t}=0.548 \\
\mathrm{df}=17.920\end{array}$ \\
\hline $0 \mathrm{~cm} \mathrm{~B}$ & - & - & - & - & $\begin{array}{l}\mathrm{p}<0.001 \\
\mathrm{t}=-15.057 \\
\mathrm{df}=14.125\end{array}$ & $\begin{array}{l}\mathrm{p}<0.001 \\
\mathrm{t}=-14.561 \\
\mathrm{df}=15.680\end{array}$ \\
\hline $5 \mathrm{~cm} \mathrm{~B}$ & - & - & - & - & - & $\begin{array}{l}\mathrm{p}=0.777 \\
\mathrm{t}=-0.287 \\
\mathrm{df}=17.469\end{array}$ \\
\hline $10 \mathrm{~cm} \mathrm{~B}$ & - & - & - & - & - & - \\
\hline
\end{tabular}

${ }^{1} \mathrm{p}<0.001$ = estadísticamente significativo, $\mathrm{p}>0.01$ = estadísticamente no significativo, los resultados de la prueba de normalidad de Shapiro-Wilk mostraron en parte una distribución normal y en parte una distribución no normal, por lo tanto se realizaron tanto la prueba U de Mann-Whitney (no paramétrica, 2 muestras independientes) como la T de significancia (paramétrica, muestras independientes); $t$ y df indican los grados de libertad dependiendo del método estadístico. 
Tabla 3. Evaluación de la emergencia de las semillas y del crecimiento de plántulas de Krameria lappacea.

\begin{tabular}{|c|c|c|c|c|c|c|c|c|c|c|c|c|}
\hline & & \multicolumn{10}{|c|}{ Hoyo } & \multirow[b]{2}{*}{$\%$} \\
\hline & & 1 & 2 & 3 & 4 & 5 & 6 & 7 & 8 & 9 & 10 & \\
\hline Fecha & & \multicolumn{11}{|c|}{ Resiembra y emergencia de semillas } \\
\hline $11 / 2011$ & Resiembra & 10 & 10 & 10 & 10 & 10 & 10 & 10 & 10 & 10 & 10 & 100 \\
\hline 04/2012 & Emergencia & 7 & 5 & 8 & 7 & 6 & 8 & 7 & 5 & 6 & 7 & 66 \\
\hline $11 / 2012$ & Sobrevivencia & 3 & 2 & 5 & 3 & 3 & 4 & 3 & 2 & 2 & 3 & 30 \\
\hline \multicolumn{13}{|c|}{ Crecimiento de promedio $[\mathrm{cm}]$} \\
\hline $11 / 2012$ & Crecimiento & 5 & 4 & 5 & 4 & 3 & 4 & 5 & 3 & 4 & 5 & \\
\hline $04 / 2013$ & Crecimiento & 10 & 7 & 9 & 10 & 7 & 8 & 10 & 6 & 8 & 9 & \\
\hline \multirow{2}{*}{$04 / 2014$} & Crecimiento & 23 & 21 & 20 & 20 & 19 & 20 & 20 & 19 & 17 & 18 & \\
\hline & Floración & + & + & + & + & + & + & + & + & + & + & \\
\hline \multirow{2}{*}{$04 / 2015$} & Crecimiento & 32 & 35 & 36 & 34 & 32 & 33 & 35 & 36 & 37 & 35 & \\
\hline & Floración & + & + & + & + & + & + & + & + & + & + & \\
\hline
\end{tabular}

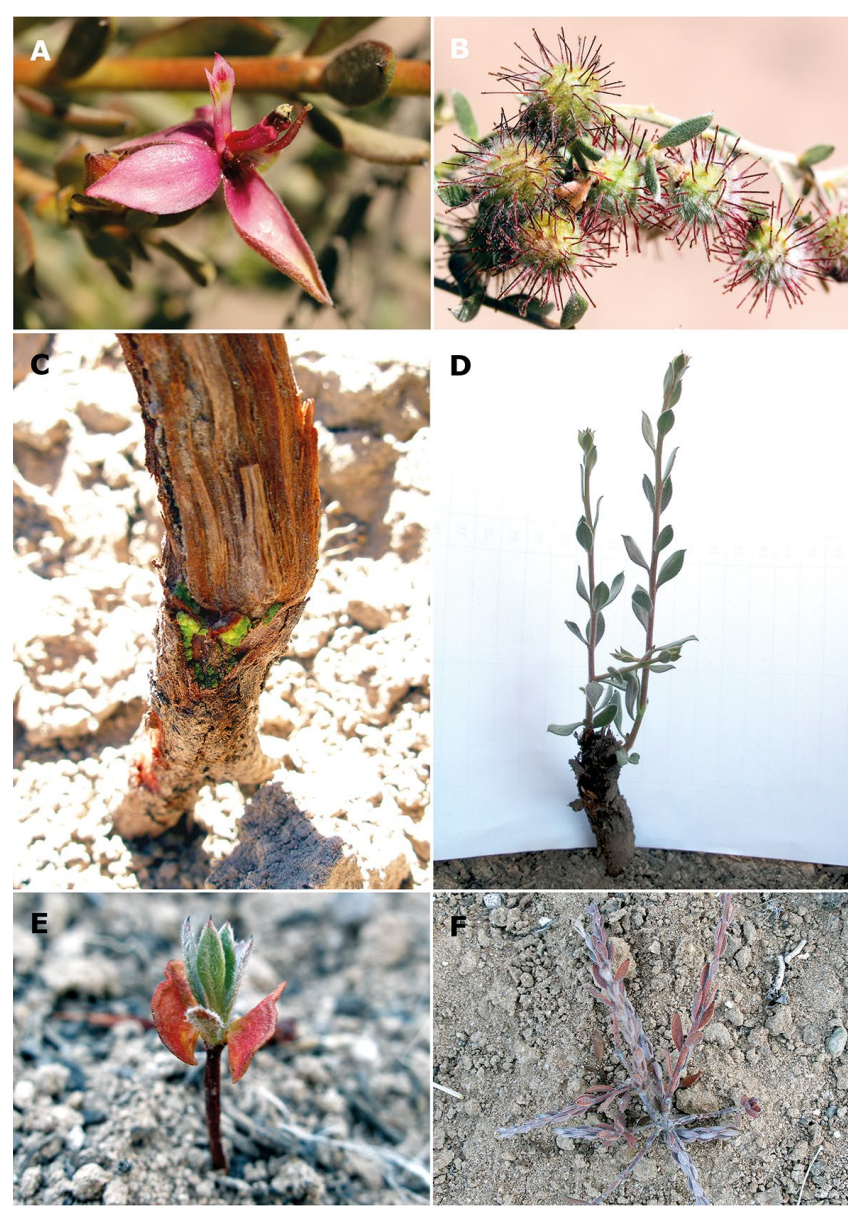

Figura 2. Krameria lappacea, A)flor; B) semilla; C) formación del meristemo de crecimiento a partir del cambium de una raíz secundaria; E+F) plántula en la resiembra superficial.

del pre-tratamiento de las semillas (escarificación) en el comportamiento de la germinación. Para lograr esto, las semillas fueron frotadas con arena gruesa con el fin de eliminar gran parte de los pelos gloquidiados y raspar el pericarpio de la semilla. Este experimento más realista no confirma el efecto positivo del pre-tratamiento de las semillas en la germinación como había sido determinado en los experimentos de laboratorio. Esto pudo deberse al hecho de que en los experimentos de laboratorio la cubierta de las semillas fue quebrada con un martillo, lo cual fue mucho más eficiente para lesionar las semillas. Sin embargo, es notable que incluso mediante dicho tratamiento únicamente se alcanzaron tasas de germinación del $62 \%$.

Los resultados de estos experimentos no tienen consecuencias directas en el manejo in situ de $K$. lappacea. En la práctica de la recolecta silvestre sostenible, las semillas serán resembradas en los hoyos resultantes de la cosecha, sin pre-tratamiento, tal y como quedan bajo las plantas recolectadas. En este caso se toma en cuenta las recomendaciones realizadas en el manejo sustentable de ratania (Weigend M. \& N. Dostert 2008) Por lo tanto el tratamiento previo posiblemente puede ser omitido, incluso en la resiembra de semillas esparcidas sobre el suelo debido a la falta de efectos positivos consistentes en la tasa de germinación. En los experimentos de resiembra esparcida sobre el suelo se demostró el éxito de la germinación sin que las semillas hubieran sido previamente tratadas. Sin embargo, la remoción de los pelos gloquidiados ayuda en la separación y limpieza las semillas, que de una u otra manera son difíciles de manipular ya que forman conglomerados en los que las semillas muertas o dañadas son difícilmente reconocibles e imposibles de separar. En consecuencia, se recomienda un tratamiento previo mediante el frote con arena gruesa para obtener semillas completamente maduras y fáciles de manipular, que puedan ser contados y resembrados en los hoyos.

Los experimentos de invernadero han confirmado la hipótesis según la cual el seed burial es un importante factor limitante en la germinación de Krameria como en varias especies de plantas de hábitats áridos (Beck 2010, Ren 2002). Según dicha hipótesis, la tasa de germinación puede incrementarse significativamente si las semillas son enterradas $(3-4 \mathrm{x})$. Sin embargo, la profundidad exacta a la cual las semillas son sembradas parece jugar un papel bastante secundario. Probablemente la profundidad de siembra adecuada se encuentra entre los $5-10 \mathrm{~cm}$. Una profundidad mucho mayor puede, por el contrario, tener un efecto negativo sobre la germinación.

Aunque el rebrote de las raíces secundarias pudo ser documentado, es decir, la formación de plántulas que nacen a partir de las raíces, no es posible asegurar que durante la práctica de la cosecha se obtengan raíces secundarias individuales con haustorios intactos a partir de cada planta cosechada. Aunque las raíces secundarias a menudo crecen perpendicularmente respecto a la raíz principal y paralelas a la superficie del suelo, las conexiones de las raíces con la planta hospedera pueden ser estropeadas debido a los movimientos de la tierra. Sin embargo, la preservación de las raíces secundarias individuales puede de 
todos modos contribuir a la regeneración natural dentro del marco de la explotación de las poblaciones de ratania.

\section{Conclusiones}

1. En cuanto a la evaluación sobre la viabilidad de las semillas de $K$. lappacea se demostró que el $75 \%$ de las semillas tratadas con la solución de trifeniltetrazol $(0.1 \%)$ fueron viables y el $25 \%$ resultaron ser inviables. La tasa de imbibición fue del $95 \%$ y de germinación del $62 \%$ después de 21 días a través de la escarificación de las semillas. Por lo tanto los experimentos de invernadero confirman la hipótesis según la cual el seed burial es un importante factor limitante en la germinación y la propagación natural de Krameria.

2. En cuanto a la evaluación de emergencia de semillas y crecimiento de las plántulas in situ, las pruebas de siembra en las localidades silvestres mostraron que 66 de las 100 semillas aportadas germinaron después de la primera época de lluvia de las cuales 30 sobrevivieron al siguiente periodo seco, logrando establecerse en la localidad silvestre, al tercer año por lo que una resiembra de semillas durante la recolección puede facilitar el establecimiento de plantas nuevas in situ y dar lugar a la regeneración natural.

3. Los resultados de este estudio apoyan las acciones propuestas en el plan de manejo para mejorar la regeneración natural de $K$. lappacea (Weigend M. \& Dostert N. 2008). Tanto la resiembra de las semillas bajo tierra como el abandono de raíces secundarias sobre el suelo y la resiembra extensiva de semillas esparcidas sobre el suelo pueden contribuir a la regeneración natural de las poblaciones silvestres de ratania.

\section{Agradecimientos}

Agradecemos a WELEDA AG Arlesheim, Suiza, y la Sociedad Alemana de Cooperación Internacional (GIZ) GmbH, Eschborn, Alemania, por su apoyo en el marco de cooperación interinstitucional público-privada. Agradecemos también a A. Ellenberger y C. Pfisterer (WELEDA AG) por su continuo apoyo a este proyecto y a Luis Martinez Manchego por la ayuda con la evaluación de la viabilidad y germinación.

\section{Literatura citada}

Adwan G., Y. Salameh, K. Adwan \& A. Barakat. 2012. Assessment of antifungal activity of herbal and conventional toothpastes against clinical isolates of Candida albicans. Asian Pacific Journal of Tropical Biomedicine 2: 375-379. https://doi. org/10.1016/S2221-1691(12)60059-8
Beck M.J. \& S.B. Vander Wall. 2010. Seed dispersal by scatterhoarding rodents in arid environments. Journal of Ecology 98: 1300-1309. http://dx.doi.org/10.1111/j.13652745.2010.01716.x

Brokamp G., N. Dostert, F. Cáceres \& M. Weigend. 2012. Parasitism and haustorium anatomy of Krameria lappacea (Dombey) Burdet \& BB Simpson (Krameriaceae), an endangered medicinal plant from the Andean deserts. Journal of Arid Environments, 83, 94-100. https://doi.org/10.1016/j. jaridenv.2012.03.004

Brokamp G. 2015. Relevance and Sustainability of Wild Plant Collection in NW South America. Springer Fachmedien Wiesbaden, Alemania. 103-122.

Cannon W.A. 1910. The root habits and parasitism of Krameria canescens Gray. In D.T. Macdougal and W. A. Cannon. The conditions of parasitism in plants. Publ. Carnegie Inst. Wash. 129: 1-60.

Carini M., G. Aldini, M. Orioli \& R.M. Facino. 2002. Antioxidant and photoprotective activity of a lipophilic extract containing neolignans from Krameria triandra roots. Planta Med. 68(3):193-7. https://doi.org/10.1055/s-2002-23167

Kuijt J. 1969. The biology of parasitic flowering plants. University of California Press, Berkerly, California.

Musselman L. J. 1975. Parasitism and haustorial structure in Krameria lanceolata (Krameriaceae). A preliminary study. Phytomorphology 25: 416-422.

Ren J., L. Tao \& X.-M. Liuz. 2002. Effect of sand burial depth on seed germination and seedling emergence of Calligonum L. species. Journal of Arid Environments 51: 603-611. https:// doi.org/10.1006/jare.2001.0979

Scholz E. \& H. Rimpler. 1989. Proanthocyanidins from Krameria triandra root. Planta Med. 55(4):379-384. https://doi. org/10.1055/s-2006-962032

Simpson B.B. 1982. Krameria (Krameriaceae) flowers: orientation and elaiophore morphology. Taxon 31: 517-528. https:// doi.org/10.2307/1220683

Simpson B.B. 1989. Krameriaceae. In Flora Neotropica. The New York Botanical Garden. Monograph 49.

Simpson B.B. 1991. The Past and Present Uses of Rhatany (Krameria, Krameriaceae). Economic Botany 45(3): 397-409. https:// doi.org/10.1007/BF02887080

Simpson B.B. 2004. Krameriaceae (Rhatany family). In: Smith, N. P., Heald, S. V., Henderson, A., Mori, S. A. \& Stevenson, D. W. (eds.): Flowering Plants of the Neotropics. Princeton University Press/New York Botanical Garden Press. 108-200.

Simpson B.B. 2007. Krameriaceae, In: Kubitzki, K., (ed.), The Families and Genera of Vascular Plants IX, 208-212.

Weigend M. \& N. Dostert. 2005. Towards a standardization of biological sustainability: Wildcrafting Rhatany (Krameria lappacea) in Peru. Medicinal Plant Conservation 11:24-27.

Weigend M. \& N. Dostert. 2008. Manejo sustentable de ratania en Perú - Krameria lappacea (Dombey) Burdet \& Simpson. botconsult $\mathrm{GmbH}$, Berlin, Alemania. 